\title{
Gynaecological Cancer
}

\section{A Tertiary Cancer Center Experience of 52 Cases of Primary Ovarian Mucinous Adenocarcinomas}

\author{
Gaurav Das ${ }^{1} \quad$ V. Sridevi ${ }^{1,2} \quad$ Mohanaraj Natarajan ${ }^{1}$ \\ ${ }^{1}$ Department of Surgical Oncology, Cancer Institute (WIA), Chennai, \\ Tamil Nadu, India \\ ${ }^{2}$ Division of Gynecologic Oncology, Department of Surgical \\ Oncology, Cancer Institute (WIA), Chennai, Tamil Nadu, India
}

\begin{abstract}
Address for correspondence Gaurav Das, MS (Surgery), MCh (Surgical Oncology) Department of Surgical Oncology, Cancer Institute (WIA), Sardar Patel Road, Adyar, Chennai 600020, Tamil Nadu, India (e-mail: das.drgaurav@gmail.com).
\end{abstract}

South Asian J Cancer:2020;9:90-92

\begin{abstract}
Keywords

- cytoreductive surgery

- mucinous adenocarcinoma

- neoadjuvant chemotherapy

- ovarian cancer

\section{Introduction}

Primary mucinous epithelial ovarian adenocarcinoma (mEOC) constitutes a small percentage (2-5\%) of ovarian cancers, with distinct clinicopathological characteristics when compared with the more common serous histology. ${ }^{1,2}$ The representation of $\mathrm{mEOC}$ in large clinical trials exploring the role of therapeutic interventions is small; for example, $1.6 \%$ in the Gynecologic Oncology Group (GOG) 182 trial. ${ }^{3}$ This subset of tumors has a low response to primary chemotherapy. There is no gold standard method to distinguish unequivocally between $\mathrm{MEOC}$ and mucinous carcinomas metastatic to the ovary.
\end{abstract}

Background Primary mucinous epithelial ovarian adenocarcinoma (mEOC) constitutes a small percentage (2-5\%) of ovarian cancer. Our aim is to understand the clinicopathological characteristics and survival results of patients with $\mathrm{mEOC}$ after a long-term follow-up.

Materials and Methods This is a retrospective study of primary mEOC cases treated at a tertiary cancer center in India, from January 1, 2005, to December 31, 2012.

Results Out of 958 malignant ovarian tumors, 52 (5.43\%) were mucinous adenocarcinoma. Nearly $71.2 \%$ of cases were of early-stage disease, and the remaining were of advanced-stage disease. After a follow-up period of 63 months (range: 1-138 months), the 5-year actuarial overall survival for stages I, II, III, and IV was 92.5, 70, 38.5, and 0\%, respectively. Among advanced-stage tumors, half of them progressed without undergoing cytoreductive surgery and died.

Conclusion Most of the mEOC cases present in earlystages and have good clinical outcome. Patients with advanced-stage disease do not respond well to standard chemotherapy regimens in use and have poor survival figures. The use of primary cytoreduction should be considered in the place of interval cytoreduction for advanced mEOC.

\section{Materials and Methods}

This is a retrospective study of a series of primary $\mathrm{mEOC}$ diagnosed and treated at a premier tertiary care center in India, from January 1, 2005, to December 31, 2012. The archived case records were studied in detail, including the clinical history and examination, imaging, endoscopy, biopsy, and histopathological reports. The following parameters were collected: age, date of admission, presenting complaints, menopausal status, parity, laterality, size of the tumor, stage of disease, serum tumor markers (cancer antigen 125 [CA-125] and carcinoembryonic antigen), use of neoadjuvant chemotherapy (NACT), date of surgery, presence of malignant ascites, peritoneal disease, whether appendicectomy was done or not and the histopathological finding of the appendix, lymph nodal dissection and the nodal status, pathological stage, grade of tumor, adjuvant chemotherapy, recurrence, date of last follow-up, and status at follow-up. Data were analyzed using simple statistical tools such as median values and SPSS version 22.0 (IBM Corp., Armonk, New York, USA). KaplanMeier plots were used to estimate the survival distribution.
Dol https://doi.org/

10.1055/s-0040-1721211

ISSN 2278-330X. (c) 2020. MedIntel Services Pvt Ltd.

This is an open access article published by Thieme under the terms of the Creative Commons Attribution-NonDerivative-NonCommercial-License, permitting copying and reproduction so long as the original work is given appropriate credit. Contents may not be used for commercial purposes, or adapted, remixed, transformed or built upon. (https://creativecommons.org/licenses/by-nc-nd/4.0/)

Thieme Medical and Scientific Publishers Pvt. Ltd., A-12, 2nd Floor, Sector 2, Noida-201301 UP, India 


\section{Results}

A total of 958 malignant ovarian tumors were treated at our institute during this period, out of which 758 were malignant epithelial ovarian cancers, 98 were germ cell cancers, 30 were sex cord stromal tumors, and 48 were not classified.

Mucinous ovarian adenocarcinomas constituted $52(5.43 \%)$ cases. Thirty-seven (71.2\%) patients presented with early-stage disease, out of which 27 (51.9\%) were in stage I and 10 (19.3\%) were in stage II. Advanced-stage disease comprised 15 (28.8\%) patients, of whom 13 (25\%) were in stage III and $2(3.8 \%)$ were in stage IV ( - Table $\mathbf{1})$.

The most common presenting complaints were abdominal distension and pain. The distribution was almost equal between premenopausal and postmenopausal women. Among the patients with early-stage tumors, $86.5 \%$ underwent primary surgery, whereas $13.5 \%$ received NACT. Stage IC and II tumors received adjuvant chemotherapy. Nearly $80 \%$ of the patients with advanced-stage disease received NACT, whereas the remainder $20 \%$ underwent primary cytoreduction. It was observed that among the 12 patients with advanced-stage disease who received NACT, 6 (50\%) progressed to chemotherapy and died without having cytoreductive surgery. One patient had secondary cytoreduction. The chemotherapy regimens used were paclitaxel and carboplatin doublet, single-agent carboplatin, cisplatin and cyclophosphamide doublet, and liposomal doxorubicin and carboplatin doublet for six cycles.

The median duration of follow-up was 63 months (range: 1-138 months). The 5 -year actuarial overall survival (OS) for stages I, II, III, and IV disease was found to be $92.5,70,38.5$, and $0 \%$, respectively ( - Table 2 ).

The age of the patient was found to be important in relation to the prognosis for the disease. For the age group 60 years or more, the 5 -year OS was $33.3 \%$ as opposed to $75.9 \%$ for those aged $<60$ years $(p=0.004)$. When the patients aged under 30 years were considered, they were found to have a 5-year and 10 -year OS of 100 and $100 \%$ as compared with 64.1 and $48.3 \%$, respectively, for those aged 30 years or more $(p=0.01)$.

Two patients who underwent fertility-preserving surgery were alive without any evidence of disease at 97 and 41 months of follow-up, respectively. One of them delivered

Table 1 The results of the various clinical characteristics studied

\begin{tabular}{|l|l|}
\hline Characteristics & Values \\
\hline Median age at presentation (years) & $45(15-73)$ \\
\hline For early-stage disease (years) & $42(15-65)$ \\
\hline For advanced-stage disease (years) & $46(36-73)$ \\
\hline Medium serum CA-125 levels (IU/mL) & 52.5 \\
\hline For early-stage disease (IU/mL) & $47.1(3.7-2,700)$ \\
\hline For advanced-stage disease $(\mathrm{IU} / \mathrm{mL})$ & $87.5(20.7-8,490)$ \\
\hline Median size of tumors $(\mathrm{cm})$ & $20(6-30)$ \\
\hline For early-stage disease $(\mathrm{cm})$ & $20(6-30)$ \\
\hline For advanced-stage disease $(\mathrm{cm})$ & $24(9.9-33)$ \\
\hline
\end{tabular}

Abbreviation: CA-125, cancer antigen 125. a healthy baby, whereas the other patient had polycystic ovarian disease and did not conceive. No statistically significant difference was observed in the survival outcome based on whether the serum CA-125 level was elevated or normal. The tumors that were $>13 \mathrm{~cm}$ and those that were unilateral fared much better than those $<13 \mathrm{~cm}$ and bilateral tumors or in cases where the laterality remained undetermined. Grade 1 and 2 tumors had better survival than grade 3 tumors $(p=0.03)(-$ Table 3$)$.

\section{Discussion}

The population-based Madras Metropolitan Tumour Registry covers 4.65 million people. The age-standardized ratio for carcinoma ovary per 100,000 population in Chennai is 8.2 as compared with 6.1 in the world. ${ }^{4}$

Mucinous adenocarcinomas of ovary are more often diagnosed at an early stage due to which the OS is better compared with that of serous carcinomas. ${ }^{5}$ In this study, $71.2 \%$ of tumors were in the early stages of disease, with a 5 -year OS of $86.2 \%$. In contrast, when the disease presents at an advanced stage, the survival is worse, probably owing to lesser sensitivity to platinum-based chemotherapy. ${ }^{2,6}$ In our study, the patients with advanced-stage disease had a 5 -year OS of $28 \%$.

The mucinous histology is uncommon. ${ }^{2}$ In our study, over a period of 8 years, only 52 cases were found comprising $5.43 \%$ of the total cases. This makes randomized controlled trials of adequate statistical power exploring therapeutic options, such as chemotherapy regimens, a daunting task.

There is no foolproof method to distinguish between primary mucinous adenocarcinomas of the ovary and mucinous adenocarcinomas metastatic to the ovary, although many

Table 2 The survival outcomes in the early and advanced stages

\begin{tabular}{|l|l|l|l|}
\hline Stage group & 3-y OS (\%) & 5-y OS (\%) & $\begin{array}{l}10-y \text { OS } \\
(\%)\end{array}$ \\
\hline Early (I and II) & 89.2 & 86.2 & 69.0 \\
\hline Advanced (III and IV) & 33.3 & 28.0 & 20.0 \\
\hline
\end{tabular}

Abbreviation: OS, overall survival.

Table 3 Survival outcome for various subgroups based on tumor characteristics

\begin{tabular}{|l|l|}
\hline Subgroup & 5 -y OS (\%) \\
\hline$<13 \mathrm{~cm}$ & 74.8 \\
\hline$>13 \mathrm{~cm}$ & 33.3 \\
\hline Unilateral & 80.5 \\
\hline Bilateral & 60.0 \\
\hline Undetermined & 0 \\
\hline Grade 1 & 75.0 \\
\hline Grade 2 & 84.0 \\
\hline Grade 3 & 41.7 \\
\hline
\end{tabular}

Abbreviation: OS, overall survival. 
algorithms using size and laterality as criteria and several immunohistochemical markers have been used over time to aid in the distinction..$^{17-9}$ These are in addition to the several morphological characteristics, such as surface involvement of ovary, a nodular growth pattern, presence of signet ring cells, hilar involvement, an expansile or infiltrative growth pattern, presence of complex papillae, and necrotic luminal debris, which have been described to make the differentiation. ${ }^{10}$

In our study, the mucinous adenocarcinomas have been deemed to have primarily arisen from the ovary based on the clinical history and examination, imaging and endoscopy, and histological features of the tumor.

mEOC responds poorly to platinum-based chemotherapy compared with its serous counterparts. ${ }^{2}$ In our study, it was observed that $6(50 \%)$ out of the 12 patients with advanced-stage disease, who received NACT with the standard platinum-containing regimens, progressed and died due to disease without getting cytoreductive surgery. This probably makes us understand that the mucinous ovarian adenocarcinomas can be considered for upfront complete cytoreductive surgery. However, such a comparison has not been made in our retrospective study, in which one-fifth of the advanced-stage $\mathrm{MEOC}$ underwent primary cytoreduction as opposed to the remaining $80 \%$ receiving NACT.

We understand that there are certain drawbacks to our study. Immunohistochemical studies were not performed, even though it was understood that they could have helped in differentiating between the primary and secondary tumors. This was because the use of these tests has several limitations and does not completely eliminate misclassification. Besides, Zaino et al reported that the median survival did not differ significantly between the groups interpreted as primary or metastatic to the ovary for stage III and IV disease. ${ }^{11}$ We have also not compared the mucinous ovarian adenocarcinomas head-on with the other histologies for the same study period.

\section{Conclusion}

Most of the mEOCs present in early stages and have good clinical outcomes. Patients with advanced-stage disease do not respond well to standard chemotherapy regimens in use and have poor survival figures. The use of complete primary cytoreduction should be considered strongly in the place of interval cytoreduction for advanced $\mathrm{MEOC}$

\section{Funding}

None.

\section{Conflicts of Interest}

There are no conflicts of interest.

\section{References}

1 Seidman JD, Kurman RJ, Ronnett BM. Primary and metastatic mucinous adenocarcinomas in the ovaries: incidence in routine practice with a new approach to improve intraoperative diagnosis. Am J Surg Pathol 2003;27:985-993

2 Shimada M, Kigawa J, Ohishi Y, et al. Clinicopathological characteristics of mucinous adenocarcinoma of the ovary. Gynecol Oncol 2009;113:331-334

3 Bookman MA, Brady MF, McGuire WP, et al Evaluation of new platinum-based treatment regimens in advanced-stage ovarian cancer: a Phase III Trial of the Gynecologic Cancer Intergroup J Clin Oncol 2009;27:1419-1425

4 Swaminathan R. Population Based Cancer Registry, Chennai. Available at: https://ncdirindia.org/Reports.aspx

5 Seidman JD, Horkayne-Szakaly I, Haiba M, et al. The histologic type and stage distribution of ovarian carcinomas of surface epithelial origin. Int J Gynecol Pathol 2004;23:41-44

6 Hess V, A'Hern R, Nasiri N, et al. Mucinous epithelial ovarian cancer: a separate entity requiring specific treatment. J Clin Oncol 2004;22:1040-1044

7 Hart WR. Diagnostic challenge of secondary (metastatic) ovarian tumors simulating primary endometrioid and mucinous neoplasms. Pathol Int 2005;55:231-243

$8 \mathrm{JiH}$, Isacson C, Seidman JD, Kurman RJ, Ronnett BM. Cytokeratins 7 and 20, dpc4, and MUC5AC in the distinction of metastatic mucinous carcinomas in the ovary from primary ovarian mucinous tumors: Dpc4 assists in identifying metastatic pancreatic carcinomas. Int J Gynecol Pathol 2002;21:391-400

9 McCluggage WG, Young RH. Immunohistochemistry as a diagnostic aid in the evaluation of ovarian tumors. Semin Diagn Pathol 2005;22:3-32

10 Lee KR, Young RH. The distinction between primary and metastatic mucinous carcinomas of the ovary: Gross and histologic findings in 50 cases. Am J Surg Pathol 2003;27:281-292

11 Zaino RJ, Brady MF, Lele SM, et al. Advanced stage mucinous adenocarcinoma of the ovary is both rare and highly lethal: a gynecologic oncology group study. Cancer 2011;117:554-562 